\title{
Der Verfassungssenat in Costa Rica - Beispiel für eine erfolgreiche Verfassungsrechtsprechung in Lateinamerika
}

\author{
Von Norbert Lösing
}

1989 wurde innerhalb des Obersten Gerichtshofs in San José ein Verfassungssenat eingerichtet $^{1}$. Damit wurde der Weg für eine konzentrierte und spezialisierte Verfassungsrechtsprechung geebnet und ein wichtiger Fortschritt in der Rechtsstaatlichkeit Costa Ricas getan. Die "Senatslösung" wurde der Gründung eines eigenständigen Verfassungsgerichts vorgezogen. Ein Grund für diese Entscheidung waren die Bedenken, ein eigenständiges Verfassungsgericht könnte zu einem Kompetenzstreit mit dem Obersten Gerichtshof führen und so eine Spaltung der judikativen Gewalt bewirken. Für die Einrichtung eines Verfassungssenats waren zudem nur geringe Reformen der Verfassung vom 7. November 1949 notwendig, die eine der stabilsten und langlebigsten Verfassungen Lateinamerikas ist. Seine ausdrückliche Grundlage in der Verfassung findet der Senat in dem neu eingeführten Art. 10: "Es obliegt einem spezialisierten Verfassungssenat des Obersten Gerichtshofs, mit absoluter Mehrheit seiner Mitglieder über die Verfassungswidrigkeit von Gesetzen jedweder Natur sowie von Akten des öffentlichen Rechts zu entscheiden..." ${ }^{2}$.

Die Regelungen zur rechtsprechenden Gewalt befinden sich im Titel XI der Verfassung. Gemäß Art. 152 wird sie durch den Obersten Gerichtshof und durch die anderen vom Gesetz vorgesehenen Gerichte ausgeübt ${ }^{3}$. Darüber hinaus bestimmt Art. 157, daß sich der Oberste Gerichtshof aus der notwendigen Anzahl Richter zusammensetzt, um eine rei-

Ley No. 7135 vom 11. Oktober 1989.

Art. 10 de la Constitución Política de la República de Costa Rica: "Corresponderá a una Sala especializada de la Corte Suprema de Justicia declarar, por mayoría absoluta de sus miembros, la inconstitucionalidad de las normas de cualquier naturaleza y de los actos sujetos al Derecho Público. No serán impugnables en esta vía los actos jurisdiccionales del Poder Judicial, la declaratoria de elección que haga el Tribunal Supremo de Elecciones y los demás que determine la ley.

Le corresponderá además:

a) Dirimir los conflictos de competencia entre los Poderes del Estado, incluido el Tribunal Supremo de Elecciones, además con las demás entidades u órganos que indique la ley.

b) Conocer de las consultas sobre proyectos de reforma constitucional, de aprobación de convenios y tratados internacionales y de otros proyectos de ley, según se disponga en la ley.

Art. 152 CCR: "El Poder Judicial se ejerce por la Corte Suprema de Justicia y por los demás tribunales que establezca la ley". 
bungslose Funktion zu garantieren und sich in die durch Gesetz vorgesehene Anzahl von Senaten unterteilt ${ }^{4}$.

Mit dem Gesetz zur Verfassungsgerichtsbarkeit vom 19. Oktober 1989 (Ley de la Jurisdicción Constitucional, LJC) werden Zusammensetzung, Verfahren und Kompetenzen des Verfassungssenats konkretisiert. Ab diesem Zeitpunkt beginnt in Costa Rica das, was viele Autoren eine "Revolution der Verfassungsrechtsprechung" genannt haben ${ }^{5}$. Tatsächlich handelt es sich bei dem Senat um den ersten Spruchkörper in Lateinamerika, der dem europäischen Modell der konzentrierten und spezialisierten Verfassungsrechtsprechung folgt ${ }^{6}$. Er wird daher nicht nur in Zentralamerika genau beobachtet, und die Übernahme eines ähnlichen Modells wird z.Zt. in Venezuela, Bolivien und Paraguay diskutiert.

\section{A. Geschichtliche Entwicklung der Verfassungskontrolle in Costa Rica und aktuelle Tendenzen}

Die Entwicklung der Verfassungskontrolle in Costa Rica läßt sich grob in vier Abschnitte unterteilen: Die Geltung der Constitución de Cádiz in der Kolonialzeit (1812-1821), die erste Phase nach der Unabhängigkeit, in der eine Verfassungskontrolle entweder gar nicht oder ausschließlich durch die Legislative durchgeführt wurde (1821-1838), ein darauffolgender Zeitraum in dem sowohl eine Kontrolle durch die Legislative als auch durch die Judikative vorgesehen war (1839-1887) und letztendlich seit 1888 der vierte Abschnitt, in dem die Verfassungskontrolle ausschließlich durch die Gerichte erfolgte, zunächst diffus,

Art. 157 CCR: "La Corte Suprema de Justicia estará formada por los Magistrados que fueren necesarios para el buen servicio, serán elegidos por la Asamblea Legislativa, la cual integrará las diversas Salas que indique la ley. La disminución del número de Magistrados, cualquiera que éste llegue a ser, sólo podrá acordarse previos todos los trámites dispuestos para las reformas parciales a la Constitución."

G. Armijo Sancho: "La Sala Constitucional: ¿Atenta conra la jurisdicción penal?", in: Ciencias Penales (Revista de la Asociación de Ciencias Penales de Costa Rica, Año 3, No 4, Junio 1991, S.21 ff (25); C.J. Gutiérrez: "Evolución de la Justicia Constitucional en Costa Rica", in: La Jurisdicción Constitucional, Seminarveröf fentlichung zum 3-jährigen Bestehen der Sala Cuarta, San José 1993, S. $171 \mathrm{ff}$ (204); M. Abellán Rosado, L. F. Cervantes Gamboa, I. González Campos, C. Zapata Calvo: "Los Efectos de las Sentencias dictadas por la Sala Constitucional en Materia de Recursos de Habeas Corpus, Amparo y Acciones de Inconstitucionalidad, Octubre 1989 a Julio 1992", Seminario de Graduación para optar al título de Licenciados en Derecho, Facultad de Derecho de la Universidad de Costa Rica, 1993, S. 4; J.F. Sáenz Carbonell: "Orígenes del Control de Constitucionalidad en Costa Rica (1812-1937)", in: Revista de Derecho Constitucional, No. 1, Enero-Abril 1991, S. 27 ff (27).

6 Die anderen verfassungsrechtsprechenden Gerichte in Lateinamerika folgen entweder dem diffusen Verfassungskontrollsystem nach nordamerikanischem Muster (z.B. der Oberste Gerichtshof in Argentinien) oder einem gemischten System mit Elementen der spezialisierten und konzentrierten sowie der diffusen Verfassungskontrolle (z.B. Verfassungsgerichte in Kolumbien und Guatemala). 
ab 1938 konzentriert durch den Obersten Gerichtshof und seit 1989 durch einen spezialisierten Verfassungssenat.

\section{Die spanische Verfassung von 1812 (Constitución de Cádiz)}

Die spanische Verfassung vom 19. März 1812 (Constitución de Cádiz) sah in ihrem Art. 373 eine Kontrolle der Verfassung ausschließlich durch die Legislative vor ${ }^{7}$. Eine Kontrolle der Legislative selber wäre undenkbar gewesen und hätte im krassen Gegensatz zu der damaligen Idealisierung der gesetzgebenden Gewalt gestanden ${ }^{8}$. Insgesamt kam es zu ca. 130 Verfahren wegen möglicher Verfassungsverletzungen vor dem Kongreß, in keinem der Verfahren ging es jedoch um die Verfassungsmäßigkeit eines Gesetzes. Der Nachteil eines solchen Kontrollsystems liegt auf der Hand und wird von C. J. Gutiérrez wie folgt treffend beschrieben: "Es ist, als habe man die Bewachung des Käses den Mäusen aufgetragen" ${ }^{9}$.

\section{Die erste Phase der Unabhängigkeit (1821-1838)}

Die ersten Verfassungen Costa Ricas waren insbesondere durch die Constitución de Cádiz und die Verfassung der Vereinigten Staaten von Amerika von 1776 beeinflußt ${ }^{10}$. Bei den Verfassungen vom 1. Dezember 1821, dem 19. März 1823 und dem16. Mai 1823 handelte es sich lediglich um nationale Versionen der Verfassung von Cádiz. Sie kannten zwar Verfahren zur Prüfung von Verfassungsverletzungen durch das Parlament, nicht jedoch zur Kontrolle der Verfassungsmäßigkeit von Gesetzen. Eine gerichtliche Verfassungskontrolle von Handlungen der öffentlichen Gewalt oder gar ein gerichtliches Normkontrollverfahren war diesen Verfassungen fremd.

III. Erweiterung der Verfassungskontrolle und Einbeziehung der judikativen Gewalt (1839-1887)

Langsam wuchs die Erkenntnis, daß auch die Überprüfung von Normen auf ihre Verfassungsmäßigkeit notwendig werden kann. Die häufigen Verfassungswechsel, die meist

Art. 373 der Constitución Política de la Monarquía Española vom 19. März 1812 (Constitución de Cádiz): "Las Cortes en sus primeras sesiones tomarán en consideración las infracciones de la Constitución que se les huebieren hecho presentes, para poner el conveniente remedio y hacer efectiva la responsabilidad de los que hubieren contravenido a ella".

M. Lorente Santineña: "Las Infracciones a la Constitución de 1812",Centro de Estudios Constitucionales, 1. Ausgabe, Madrid 1988, S. 24.

C.J. Gutierrez: "El subdesarrollo del Derecho Público", in: Revista de Ciencias Jurídicas No. 53, San José, May-August 1985, S. 58.

R. Hernández Valle: "El Sistema Constitucional Costarricense", in: Los Sistemas Constitucionales de Latinoamérica, Madrid 1992, S. 213 ff (214). 
parallel zu einem Regimewechsel erfolgten, stellten die Verfassungsmäßigkeit vieler Normen in Frage, die darüber hinaus von den jeweiligen Folgeregierungen in ihrer Legitimität angezweifelt wurden. Zunächst behielt sich das Parlament die Normenkontrolle vor $^{11}$, bis in der Verfassung von 1869 eine parallele Kontrolle durch den Obersten Gerichtshof eingeführt wurde $^{12}$. Die letzte Kontrolle oblag aber weiterhin dem Kongreß, dem der Gerichtshof seine Entscheidung zur "weiteren Behandlung" vorlegen mußte. Somit war in der Verfassung von 1869 zumindest ansatzweise die richterliche Kontrolle von Normen eingeführt worden. Aufgrund des Staatsstreiches vom 27. April 1870 wurde die Verfassung jedoch wieder aufgehoben, ohne da $\beta$ es auch nur ein richterliches Normprüfungsverfahren gegeben hatte.

\section{Die richterliche Verfassungskontrolle ab 1888}

Das am 29. März 1887 durch Dekret erlassene Gerichtsorganisationsgesetz (Ley Orgánica de Tribunales), welches am 10. Januar 1888 in Kraft trat, sah in seinem Art. 8 I Nr. 10 indirekt die richterliche Normenkontrolle vor, in dem es bestimmte: "Den Beamten der Justiz ist es verwehrt: ... Nr. 10.- Gesetze, Dekrete oder Regierungsbeschlüsse anzuwenden, die gegen die Verfassung verstoßen." ${ }^{13}$ Eine Änderung der Verfassung von 1871 fand nicht statt. Damit entstand ein Widerspruch zur Verfassung von 1871, die noch immer die ausschließliche Kompetenz des Kongresses zur Normenkontrolle vorsah. Zunächst entstanden keine Konflikte, da der Kongreß von seiner Zuständigkeit keinen Gebrauch machte. Die diffuse Verfassungskontrolle durch die Gerichte verlief in diesem Zeitraum völlig ungestört im Sinne des nordamerikanischen "judicial review" ${ }^{14}$. Dies änderte sich 1915, als der Kongreß im Verfahren Alfaro vs. Registrador ein Urteil des Kassationsgerichts (Tribunal de Casación) aufhob. Ab diesem Zeitpunkt übten die Gerichte äußerste Zurückhaltung bei der Normenkontrolle.

Am 27. Januar 1917 kam es zu einem Staatsstreich, und General Federico A. Tinoco Granados übernahm das später durch Wahlen bestätigte Amt des Präsidenten. Am 8. Juni des gleichen Jahres wurde eine neue Verfassung erlassen, in der weiterhin die Zuständigkeit des Kongresses zur Prüfung der Verfassungsmäßigkeit von Gesetzen erhalten

11 Einige Beispiele erwähnt J. F. Sáenz Carbonell (Fn 5).

12

Art. 135 der Verfassung vom 18. Februar 1869: "La Corte Suprema de Justicia puede suspender por mayoría absoluta de votos, por si, a pedimento de su Fiscal o de cualquier ciudadano la ejecución de las disposiciones legislativas que sean contrarias a la Constitución; debiendo someter al Congreso en su próxima reunión ordinaria sus observaciones, para que, tomándolas en consideración, resuelva definitivamente lo que convenga".

13

Dekret Nr. 11 vom 29. März 1887, Art. 8, Abs. 1, Nr. 10: "No podrán los funcionarios del orden judicial: 10.-Aplicar leyes, decretos o acuerdos gubernativos que sean contrarios a la Constitución."

14

C. J. V. Gutierrez: "La Constitución Norteamericana como Ley importada", in: Revista de Ciencias Jurídicas, San José, Nr. 61, September-Oktober 1988, S. 51. 
blieb. Gleichzeitig schrieb man jedoch die parallele Kontrollkompetenz der Gerichte erstmals in der Verfassung (und nicht nur im Gerichtsorganisationsgesetz) fest. Mit dem Sturz des Generals Tinoco im Jahre 1919 wurde die Verfassung von 1917 aufgehoben und die Verfassung von 1871 erneut in Kraft gesetzt. Mit Dekret Nr. 4 vom 29. Oktober 1919 wurden alle Gesetze und Regelungen der Regierung Tinoco durch die provisorische Regierung Aguilar, bis auf wenige Ausnahmen, für nichtig erklärt. Die Folge war eine große Rechtsunsicherheit. Diese sog. "Ley de Nulidades" war nämlich äußerst umstritten. Während einige Gerichte sie für verfassungswidrig erklärten, wandten sie andere, insb. das Kassationsgericht, als verfassungsmäßig an. In dieser Periode zeigten sich in besonderem Maße die Nachteile der diffusen Verfassungskontrolle, in der die Urteile der oberen Instanzen nicht bindend waren für die unteren Instanzen. Die dadurch entstandene Rechtsunsicherheit versuchte man zu beseitigen, indem man 1937 die konzentrierte Verfassungskontrolle einführte. Gem. Art. 962 bis 969 des Zivilverfahrensgesetzes (Código de Procedimientos Civiles) hatte nunmehr das Oberste Gericht in Plenarsitzungen über die Verfassungsmäßigkeit von Normen zu entscheiden. Wie bereits 1887 hatte die richterliche Normenkontrolle keine Grundlage in der Verfassung und war daher nicht unumstritten $^{15}$. Sie setzte sich jedoch durch und aufgrund der konzentrierten Verfassungkontrolle "beruhigte" sich die Verfassungsrechtsprechung in Costa Rica. Es gelang, die Rechtssicherheit wieder herzustellen. Trotzdem erfolgte der entscheidende Durchbruch in der Verfassungsrechtsprechung Costa Ricas im Jahre 1989, als die konzentrierte und spezialisierte Verfassungsrechtsprechung eingeführt wurde. Im Gegensatz zu den verfassungsrechtlichen Entscheidungen in vielen anderen Ländern Lateinamerikas sind die Entscheidungen des Verfassungssenates, wie Art. 13 der LJC vorschreibt ${ }^{16}$, für alle bindend und haben damit den Charakter einer wahren Normenkontrolle (sie genießen Bindung erga omnes und nicht nur inter partes). Dies ist ohne Zweifel ein entscheidender Grund für den Einfluß, den die Rechtsprechung des Senats gehabt hat und noch heute hat.

Wie der kurze geschichtliche Abriß zeigt, begann in Costa Rica bereits früh eine eigenständige Entwicklung, die zu einer Verdichtung der Verfassungskontrolle im spezialisierten Senat führte. Bei der Einrichtung des Verfassungssenats handelt es sich somit nicht um eine einfache Übernahme einer konzentrierten und spezialisierten Verfassungskontrolle nach österreichischem Muster, sondern um eine gewachsene Entwicklung, die noch nicht zu einem Stillstand gekommen ist. Seit der Einrichtung des Verfassungssenates besteht Streit über die Definition des costa-ricanischen Systems als konzentriert und

So enthielt sich ein Richter des Obersten Gerichtshofs regelmäßig bei Entscheidungen des Plenums in Verfassungssachen, weil er die besagten Vorschriften aus dem Zivilprozeßrecht für verfassungswidrig hielt; siehe C.E. Salazar G., "Procedimientos Civiles", Facultad de Derecho, Universidad de Costa Rica, ohne Datum, S. 142.

Art. 13 LJC: "La jurisprudencia y los precedentes de la jurisdicción constitucional son vinculantes erga omnes, salvo para sí misma". 
spezialisiert. Sich auf die Art. 11 und 194 der Verfassung ${ }^{17}$ berufend argumentieren verschiedene Verfassungsrechtler ${ }^{18}$, in Costa Rica herrsche das gemischte System der diffusen und konzentrierten Verfassungskontrolle. Argument ist dabei der Wortlaut der genannten Vorschriften, der Richter, Justizangestellte und andere Beamte dazu verpflichtet, die Verfassung zu achten. Gegen diese, auch von einigen Richtern des Verfassungssenates geteilte Interpretation sprach der Wortlaut des Art. 8 des Gerichtsorganisationsgesetzes $^{19}$ (alte Fassung), der es zwar allen Justizangestellten verbietet, Gesetze und Dekrete anzuwenden, die gegen die Verfassung verstoßen aber nur dann, wenn der Verstoß zuvor vom zuständigen Gericht festgestellt wurde. Nach Ansicht einiger Verfassungsrechtler ist die Hoheit der Verfassung jedoch nur dann zu wahren, wenn jeder Richter ein Gesetz, welches er für verfassungswidrig hält, im konkreten Fall außer Acht lassen kann. Die Entscheidung des Richters hat dann Geltung inter partes. Meines Erachtens ergibt sich aus den genannten Vorschriften keineswegs die Normenkontrollzuständigkeit eines jeden Richters. Insbesondere der Wortlaut des Art. 8 des Gerichtsorganisationsgesetzes (alte Fassung) ließ eine solche Interpretation nicht zu. Die neue Fassung des Art. $8^{20}$ hingegen öffnet die Tür für eine diffuse Normenkontrolle, wenn man ihn so interpretiert, daß ein Richter nur dann vorlegen muß, wenn er Zweif el über die Verfassungsmäßigkeit einer Norm hat (er hat insofern keinen Ermessensspielraum mehr). Ist er sich jedoch über die Verfassungswidrigkeit sicher, könnte er im konkreten Verfahren auch selber entscheiden und die betreffende Norm außer Acht lassen. Derzeit herrscht in Costa Rica noch die konzentrierte und spezialisierte Verfassungskontrolle. Zur Zeit ist ein Verfahren beim

Art. 11 der Verfassung: "Los funcionarios públicos son simples depositarios de la autoridad y no pueden arrogarse facultades que la ley no les concede. Deben prestar juramento de observar y cumplir esta Constitución y las leyes. La acción para exigirles la responsabilidad penal de sus actos es pública."

Art 194 der Verfassung: "El juramento que deben prestar los funcionarios públicos, según lo dispuesto en el Art. 11 de esta Constitución es el siguiente: "¿Juráis a Dios y prometéis a la Patria, observar y defender la Constitución y las leyes de la República,y cumplir fielmente los deberes de vuestro destino? - Sí juro - . Si así lo hiciéreis, Dios os ayude, y si no, El y la Patria os lo demanden".

Vgl. z.B. L.P. Mora Mora (aktueller Präsident des Verfassungssenates): "La Jurisdicción Constitucional Costaricense", in: "La Justicia Constitucional: Una Promesa de la Democracia", Tomo II, San José, 1992, S. 201 ff (214/215).

Art. 8 Abs. 1 Ley Orgánica del Poder Judicial (alte Fassung): "No podrán los funcionarios que administran justicia: 1) Aplicar leyes, decretos, acuerdos o resoluciones gubernativas que sean contrarios a la Constitución, cuando la inconstitucionalidad haya sido declarada por los Tribunales correspondientes, de acuerdo con lo que dispone el Código de Procedimientos Civiles, de una manera especial o en casos iguales al que estuviere resuelto."

Art 8 Ley Orgánica del Poder Judicial (neue Fassung): "Los funcionarios que administran justicia no podrán: 1) Aplicar leyes u otras normas o actos de cualquier naturaleza que sean contrarios a la Constitución Política.

Si tuvieren duda sobre la constitucionalidad de esas normas o actos, deberán hacer la consulta correspondiente a la jurisdicción constitucional.

Tampoco podrán interpretarlos o aplicarlos de manera contraria a los precedentes o jurisprudencia de la Sala Constitucional." 
Senat zur Überprüfung der Verfassungsmäßigkeit von Art. 8 der Ley Orgánica del Poder Judicial anhängig. Ich glaube nicht, daß die diffuse Verfassungskontrolle, wie bezweckt, zur Entlastung des Verfassungssenates beitragen würde. Sie würde im Gegenteil eher zu einer Rechtsunsicherheit führen, die gerade durch die konzentrierte Verfassungsrechtsprechung beseitigt worden war. Dies würde auch nicht durch die Bindung der Gerichte an die Rechtsprechung des Verfassungssenates vermieden werden, da diese Rechtsprechung zum Teil erst mit großer Verspätung veröffentlicht wird und daher nicht allen Richtern bekannt ist (dies beweist alleine die hohe Anzahl an abgelehnten Richtervorlagen wegen schon vorliegender Rechtsprechung). Bedenkt man weiterhin, daß Gerichtsentscheidungen gem. Art. 10 der Verfassung vom Verfassungssenat nicht überprüft werden können, würde eine Entscheidung über die Verfassungswidrigkeit von Normen z.T. auf die ordentliche und Verwaltungsgerichtsbarkeit verlagert, da auch das Rechtsmittel gegen die fragliche Entscheidung zu einem Gericht desselben Zweiges führt. Eine Überprüfung noch mit Wirkung im konkreten Fall durch den Verfassungssenat könnte die interessierte Partei nur dann erreichen, wenn man entgegen dem Wortlaut des Art. 73 der LJC die Parteienvorlage auch zur Überprüfung der Verfassungsmäßigkeit - und nicht nur der Verfassungswidrigkeit - einer Norm zuließe (sozusagen die "Acción de Inconstitucionalidad" zu einer "Acción de Constitucionalidad" macht). Meines Erachtens verstößt Art 8 des Gerichtsorganisationsgesetzes (neue Fassung) bei der oben dargelegten Interpretation zudem gegen die Verfassung, die in ihrem Art. 10 die Zuständigkeit zur Normenkontrolle ausdrücklich dem Verfassungssenat zuweist.

\section{B. Der Verfassungssenat}

Der Verfassungssenat ist Teil des Obersten Gerichtshofs und einer von vier Senaten. Er besteht aus sieben hauptamtlichen Richtern und zwölf Ersatzrichtern. Die Richter werden vom Kongreß mit Zweidrittel-Mehrheit für den Zeitraum von acht Jahren gewählt (Ersatzrichter für den Zeitraum von vier Jahren). Sie gelten für den gleichen Zeitraum als wiedergewählt, wenn sie nicht mit der gleichen Mehrheit abgewählt werden. Art. 159 der Verfassung bestimmt die Voraussetzungen für das Richteramt, die für die Besetzung des gesamten Obersten Gerichtshof gelten. Demnach müssen die Richter durch Geburt oder durch Annahme costa-ricanische Staatsangehörige sein. Solche, die die Staatsbürgerschaft angenommen haben, müssen zumindest zehn Jahre ununterbrochen im Land gewohnt haben. Sie müssen im vollen Genuß ihrer Rechte sein und dürfen keine kirchlichen Ämter innehaben. Das Mindestalter beträgt 35 Jahre. Die Richter müssen weiterhin Volljuristen (Rechtsanwälte) sein und zumindest zehn Jahre als solche in ihrem Beruf gearbeitet haben. Bei Justizangestellten reicht jedoch eine Berufserfahrung von fünf Jahren. Gem. Art. 160 der Verfassung kann derjenige nicht zum Richter des Obersten Gerichtshofes gewählt werden, der bis zum dritten Grade mit einem amtierenden Richter des Gerichtshofes verwandt ist. Die Ersatzrichter werden auch vom Kongreß gewählt aus einer vom 
Obersten Gerichtshof vorgeschlagenen Liste. Nicht ganz unproblematisch ist die Tatsache, da $\beta$ die Ersatzrichter meist praktizierende Anwälte sind, die häufig Parteien vor dem Verfassungssenat vertreten. Hier könnten sich, insbesondere bei ähnlich gelagerten Fällen, Interessenkonflikte ergeben.

Um der hohen Arbeitsbelastung Herr zu werden, zählen die Richter auf die Unterstützung von Assistenten. Dabei hat jeder Richter des Verfassungsenates zwei Assistenten, der Präsident zehn ${ }^{21}$. Weiterhin ist der Senat mit einer ausreichenden Anzahl von Schreibkräften und anderem Hilfspersonal ausgestattet. Seit ungefähr einem Jahr stehen ihm auch moderne Computeranlagen zur Verfügung. Im Gerichtsgebäude können die Richter auf eine für lateinamerikanische Verhältnisse gut ausgestattete Bibliothek zurückgreifen. Die Bezahlung der Richter ist angemessen und garantiert ihre Unabhängigkeit.

\section{Die Kompetenzen des Verfassungssenats}

Die Kompetenzen des Verfassungssenates ergeben sich aus Art. 10 der Verfassung Costa Rica's in Verbindung mit dem Verfassungsgerichtsbarkeitsgesetz (Ley de la Jurisdicción Constitucional, LJC). Die LJC überträgt vier wichtige Zuständigkeiten an den Verfassungssenat: Habeas Corpus, Verfassungsbeschwerden, Normenkontrollen und Kompetenzstreitigkeiten $^{22}$. Sowohl bei den Verfassungsbeschwerden wie auch bei den Normenkontrollen gibt es verschiedene Verfahren. In einem generalklauselartigen Absatz des Art. 2 der LJC werden weitere Kompetenzen eröffnet, soweit sie in der Verfassung oder in der LJC vorgesehen sind ${ }^{23}$. Prüfungsmaßstab für den Verfassungssenat sind sowohl die

Dies erklärt sich aus der erhöhten Arbeitsbelastung des Präsidenten (siehe Acciones de Inconstitucionalidad). Alle Assistenten sind Juristen.

Art. 2 LJC: "Le corresponde específicamente a la jurisdicción constitucional:

a) Garantizar, mediante los recursos de hábeas corpus y de amparo, los derechos y libertades consagrados en la Constitución Política y los derechos humanos reconocidos por el Derecho Internacional vigente en Costa Rica.

b) Ejercer el control de la constitucionalidad de las normas de cualquier naturaleza y de los actos sujetos al Derecho Público así como la conformidad del ordenamiento interno con el Derecho Internacional o Comunitario, mediante la acción de inconstitucionalidad y demás cuestiones de constitucionalidad.

c) Resolver los conflictos de competencia entre los Poderes del Estado, incluido el Tribunal Supremo de Elecciones, y los de competencia constitucional entre éstos y la Contraloría General de la República, las municipalidades y las demás personas del Derecho Público.

d) Conocer de los demás asuntos que la Constitución o la presente Ley le atribuyan. 
Verfassung Costa Ricas als auch die Menschenrechtsabkommen, denen Costa Rica beigetreten ist $^{24}$.

\section{Habeas Corpus}

Dieses, zu Recht als das "Ursprungsgrundrecht" bezeichnete Menschenrecht findet besondere Berücksichtigung im Rechtssystem Costa Ricas. Der Schutz vor willkürlicher Verhaftung und Strafverfolgung ist die Voraussetzung dafür, daß man überhaupt den Schutz des Gesetzes genießt, daß der Freiheitsraum berechenbar ist und da $ß$ man sich in diesem Freiheitsraum selbstsicher bewegen und behaupten kann ${ }^{25}$. Das Recht auf Beschreitung des Habeas-Corpus-Verfahrens wird in Art. 48 der Verfassung Costa Rica's zum individuellen Grundrecht erhoben ${ }^{26}$ und ist in den Art. 15 bis 28 der LJC geregelt. Diese Vorschrift verweist auf Art. 10 der Verfassung und begründet somit die Kompetenz des Verfassungssenates $^{27}$. Die Freiheit der Person ist die Grundlage der allgemeinen Rechtsstellung des Bürgers und nimmt deswegen einen hohen Rang unter den Verfassungsgarantien ein. Bereits der erste Artikel des Kapitels "Individuelle Rechte und Garantien" der Verfassung garantiert die Freiheit der Person noch vor dem Recht auf Leben ${ }^{28}$. Der Stellenwert der persönlichen Freiheit wird auch dadurch verdeutlicht, daß das früher in der Kompetenz der Strafgerichte liegende Habeas-Corpus-Verfahren nunmehr in der Kompetenz des Verfassungssenates liegt. In der LJC wird es noch vor der Verfassungsbeschwerde aufgeführt. Das Habeas-Corpus-Verfahren beruht in erster Linie auf dem angelsächsischen "Writ of Habeas Corpus", welches sich zunächst als Gewohnheitsrecht entwickelte, bevor es 1679 in ein Gesetz aufgenommen wurde. Zuvor gab es bereits einen Rechtsschutz über die "Writ de Odio et Atia" und die "Writ de Homine Repigliando", welche durch eine Verschmelzung zum "Writ of Habeas Corpus" geführt haben dürften ${ }^{29}$. Mit einem "Writ de Odio et Atia" sollte derjenige wieder in Freiheit gesetzt werden, der

Art. 1 LJC: "La presente Ley tiene como fin regular la jurisdicción constitucional, cuyo objeto es garantizar la supremacía de las normas y principios constitucionales y del Derecho Internacional o Comunitario vigente en la República, su uniforme interpretación y aplicación, así como los derechos y libertades fundamentales consagrados en la Constitución o en los instrumentos internacionales de derechos humanos vigentes en Costa Rica."

M. Kriele: "Recht, Vernunft, Wirklichkeit", Teil II, Menschenrechte, Berlin 1990, S. 88.

26

R. Hernández Valle: "La Tutela de los Derechos Fundamentales", San José, 1990, S. 38.

Art. 48 Constitución de Costa Rica: "Toda persona tiene derecho al recurso de hábeas corpus. Ambos recursos serán de la competencia de la sala indicada en el artículo 10".

Art. 20 Constitucíon de Costa Rica: "Todo hombre es libre en la República; no puede ser esclavo él que se halle bajo la protección de sus leyes".

Art. 21 CCR: "La vida humana es inviolable".

I. Burgoa O.: Diccionario de Derecho Constitucional, Garantías y Amparo, México 1992, Habeas Corpus, S.210. 
durch $\mathrm{Ha}$ oder Betrug in Haft geraten war. Durch den "Writ de Homine Repigliando" konnte ein "Sherrif" dazu gezwungen werden, einen Verhafteten vorläufig freizulassen.

Eine weitere Wurzel des lateinamerikanischen und costa-ricanischen Habeas-CorpusVerfahrens liegt in der "manifestación de las personas" oder dem "Amparo" des mittelalterlichen aragonensischen Foralrechts, welches seine Blütezeit zwischen dem 14. und dem 16. Jahrhundert erlebte ${ }^{30}$. Das in der LJC vorgesehene Habeas-Corpus-Verfahren ist jedoch weitergehend und schützt auch bei Eingriffen in und Bedrohungen der Freiheit. Hiervon mitumfaßt werden ausdrücklich auch die Rechte auf Ein- und Ausreise sowie die Aufenthalts- und Niederlassungsfreiheit innerhalb Costa Ricas ${ }^{31}$. Alle Handlungen der öffentlichen Gewalt, auch der Richter und Strafrichter (also auch Urteile) unterliegen der Kontrolle durch das Habeas-Corpus-Verfahren ${ }^{32}$.

Das Verfahren wurde in dem Bewußtsein entworfen, daß der Einzelne dem Staat in einem Unter- und Überordnungsverhältnis gegenübersteht. Dementsprechend wurde der Zugang zum Verfassungssenat stark vereinfacht und auf jedwede Formalität verzichtet. Gemäß Art. 18 LJC kann jedermann ${ }^{33}$ das Rechtsmittel des Habeas Corpus schriftlich einlegen $^{34}$. Mit dem Eingang des Schreibens wird das Verfahren vom Präsidenten des Verfassungssenates oder von einem hierfür jeweils zuständigen Richter des Senates (Magistrado instructor) eröffnet. Gemäß Art. 19 LJC ist das Verfahren vorrangig zu bearbeiten. Der zuständige Richter fordert die beschuldigte Behörde (die die Freiheitsbeeinträchtigung zu verantworten hat) auf, innerhalb einer Frist von höchstens drei Tagen einen Bericht ${ }^{35}$

H. Fix Zamudio: "Die Verfassungskontrolle in Lateinamerika", in H.R. Horn / A. Weber (Hrsg.), Richterliche Verfassungskontrolle in Lateinamerika, Spanien und Portugal, (Studien und Materialien zur Verfassungsgerichtsbarkeit Nr. 45), Baden Baden 1989, S. 129 ff. (139).

Art. 15 LJC: "Procede el hábeas corpus para garantizar la libertad e integridad personales, contra los actos $u$ omisiones que provengan de una autoridad de cualquier orden, incluso judicial, contra las amenazas a esa libertad y las perturbaciones o restricciones que respecto de ella establezcan indebidamente las autoridades, lo mismo que contra las restricciones ilegítimas del derecho de trasladarse de un lugar a otro de la República, y de libre permanencia, salida e ingreso en su territorio".

32 Besprechung der Entscheidung des Verfassungssenates 500-90 vom 15. Mai 1990, in: Revista de Derecho Constitucional Nr. 1, San José, Enero-Abril 1991, S. 165 f. (166).

Also auch jemand, der nicht selbst und unmittelbar betroffen ist. Es ist keine amtliche Beglaubigung des Dokuments nötig, und in der Praxis wird das Verfahren auch durch ein nicht unterschriebenes Rechtsmittel in Gang gesetzt. Eines der ersten Habeas-Corpus Verfahren vor dem Senat wurde durch den mit Bleistift geschriebenen und nicht unterzeichneten Brief eines kleinen Jungen eröffnet, der sich gegen die Internierung in einem Pflegeheim wehrte. Der Brief war zunächst im Papierkorb gelandet, wurde jedoch zufällig von einem der Richter gefunden.

Art. 18 LJC: "Podrá interponer el recurso de hábeas corpus cualquier persona, en memorial, telegrama u otro medio de comunicación escrito, sin necesidad de autenticación. Cuando se utilice la vía telegráfica se gozará de franquicia".

Art 22 LJC bestimmt den Mindestinhalt des angeforderten Berichts. Danach muß der Grund für die Freiheitsbeeinträchtigung erläutert werden und die rechtliche Grundlage für die getroffene Maßnahme 
über die von ihr ergriffenen und angeordneten Maßnahmen an ihn zu senden. Laut Art. 8 Abs. 4 LJC handelt es sich bei den Fristen im Rahmen des Habeas-Corpus-Verfahrens um Kalendertage. Wird die Frist nicht eingehalten, kann gemäß Art. 23 LJC die Wahrheitsvermutung der Rechtsmittelbegründung eingreifen. Dieses liegt im Ermessen der Richter. Der den Fall zunächst bearbeitende Richter kann die notwendigen Weisungen erteilen, um dem späteren Urteil des Senates auf jeden Fall Geltung zu verschaffen und alle Handlungen zu unterbinden, die den Erfolg des Rechtsmittels vereiteln könnten. Diese im Art. 19 Abs. 2 LJC geregelte Zuständigkeit des einzelnen Richters wird durch Art. 21 Abs. 3 LJC dahingehend ergänzt, daß der Senat vorläufige Maßnahmen zum Schutz des Betroffenen anordnen kann. Innerhalb von fünf Tagen nach Ablauf der im Art. 19 Abs. 2 LJC vorgesehenen Frist bzw. nach der mündlichen Verhandlung, falls eine solche durchgeführt wird, muß der Senat über das Rechtsmittel entscheiden, es sei denn es sind nach Ansicht des Senats noch weitere Beweiserhebungen erforderlich. In diesem Falle läuft die Frist von fünf Tagen erst ab dem Zeitpunkt des Eingangs der erhobenen Beweismittel. Das Erhebungsverfahren und auch die mündliche Verhandlung (soweit notwendig), sind schnellstmöglich durchzuführen.

Art. 24 LJC enthält in seinem Abs. 2 eine nicht abschließende Auflistung der Punkte, die vom Senat im Falle eines Habeas-Corpus-Verfahrens zu prüfen sind ${ }^{36}$. Neben konkreten Prüfungspunkten enthält diese Aufzählung auch eine Generalklausel (Nr. d). Die Aufzählung ergibt sich aus den zuvor bei den Strafgerichten gemachten Erfahrungen mit dem Habeas-Corpus-Verfahren und erwähnt insbesondere die Punkte, bei denen die meisten

genannt werden. Weiterhin ist gegebenenfalls eine Kopie des Haftbefehls oder der entsprechenden Weisung beizufügen. Auch die Beweise, die gegen den Betroffenen vorliegen, sind zu erläutern.

Demnach wird der Senat zunächst prüfen:

- ob die staatliche Stelle oder Behörde die die freiheitsentziehende oder -einschränkende Maßnahme vorgenommen hat, die hierzu erforderliche Zuständigkeit besitzt (Art. 24 II a LJC),

- ob die Festnahme rechtswidrig oder unter Verstoß gegen Artikel 37 der Verfassung angeordnet wurde (Art. 24 II b LJC),

- ob der Haftbefehl oder die Untersuchungshaft rechtmäßig angeordnet wurden oder ob der Freiheitsentzug auf einem rechtskräftigen Urteil beruht (Art. 24 II c LJC),

- ob, nach Erklärung eines nationalen Notstandes, sich die angeordneten Maßnahmen im Rahmen der Verfassung halten und vernünftigerweise im Zusammenhang mit der Notstandssituation stehen und damit verhältnismäßig sind (Art. 24 II ch LJC),

- ob der Freiheitsentzug oder die angeordnete Maßnahme aus einem anderen Grund rechtswidrig war (Art. 24 II d LJC),

- ob die durch das Habeas-Corpus-Verfahren geschützten Grundrechte tatsächlich bedroht oder verletzt wurden oder werden (Art. 24 II e LJC),

- ob der Betroffene rechtswidrig in Einzelhaft genommen worden ist oder ob eine rechtmäßig angeordnete Einzelhaft über dem in Artikel 44 der Verfassung vorgesehenen Zeitraum hinaus aufrecht erhalten wird (Art. 24 II $\mathrm{f} \mathrm{LJC}$ ),

- ob die Festnahme, Haft oder angeordnete Maßnahme unter gesetzlich verbotenen Umständen durchgeführt wurde oder wird (Art. 24 II g LJC),

- ob die dem Betroffenen vorgeworfene Tat in einem bereits zum Zeitpunkt der Tat existierenden Gesetz strafbewehrt ist. 
Verfahrensverletzungen eingetreten waren. Hintergrund der Aufzählung und insbesondere der Generalklausel ist das Interesse an einem möglichst umfangreichen und gründlichen Rechtsschutz.

Sollte sich die freiheitseinschränkende oder -entziehende Maßnahme als rechtswidrig erweisen, ist das Rechtsmittel begründet (Artikel 25 LJC). Mit dem Urteil werden die angegriffenen Maßnahmen aufgehoben und die Wiederherstellung der Freiheit und Rechte des Betroffenen angeordnet. Weiterhin wird die verletzende Behörde oder Institution verurteilt, den Betroffenen zu entschädigen. Der Schadensersatz muß dann im Wege des Verwaltungsvollstreckungsverfahrens eingefordert werden. Das Urteil ist den Beteiligten zuzustellen, soweit sie eine Adresse für die Zustellung angegeben haben. Eine Ausnahme der Zustellungspflicht an den Betroffenen liegt dann vor, wenn das Rechtsmittel begründet war und der Betroffene zum Zeitpunkt der Entscheidung seine vollen Freiheitsrechte wiedererlangt hat. Der wirksamen Durchsetzung seiner Entscheidungen und auch der Beschleunigung der Verfahren vor dem Verfassungssenat dienen die Art. 71 und 72 LJC. Danach kann die Strafverfolgungsbehörde denjenigen zu einer Freiheitsstrafe von drei Monaten bis zu zwei Jahren oder zur Zahlung einer Geldstrafe verurteilen, der den Anordnungen des Senates nicht Folge leistet.

Die Einfachheit des Rechtsmittels drückt sich in der hohen Zahl der Verfahren aus. Gemeinsam mit den besonders zahlreichen Amparo-Verfahren führen die Habeas-CorpusVerfahren zu einer deutlichen Überlastung des Verfassungssenates ${ }^{37}$. Beide Verfahren entsprechen ungefähr der deutschen Verfassungsbeschwerde, die auch den Großteil der Verfahren vor dem Bundesverfassungsgericht in Karlsruhe ausmacht. Man hat deshalb versucht, den Senat durch die Bestimmungen in Art. 17 und Art. 20 LJC zu entlasten. Gemäß Artikel 17 LJC muß der bearbeitende Richter das Verfahren im Falle der offensichtlichen Unzulässigkeit oder Unbegründetheit nicht weiter betreiben und direkt dem Senat zur Ablehnung vorlegen. Die Ablehnungsentscheidung muß jedoch im Plenum erfolgen, was die Zeitersparnis und Entlastung wiederum sehr relativiert. Offensichtlich unzulässige oder unbegründete oder aber offensichtlich begründete Rechtsmittel könnten auch durch zu gründende Kammern von je vier Richtern (der Präsident müßte in beiden Kammern tätig werden) abschließend beschieden werden. Sind Festgenommene einem Richter vorgeführt worden, ohne daß jedoch von diesem bereits eine Anordnung getroffen wurde, kann der bearbeitende Richter gem. Art. 20 LJC das Verfahren aussetzen und das entsprechende Gericht auffordern, die notwendigen Entscheidungen zu treffen. Zu einer wahren Entlastung des Verfassungssenates wird es jedoch erst dann kommen, wenn das Habeas-Corpus-Verfahren gemeinsam mit einer Strafprozeßrechtsreform zumindest in erster Instanz an die Strafgerichte zurückgegeben wird. Dabei könnte der Senat, nach

1990 sind 573, 1991611,1992794 und 1993916 Habeas-Corpus-Verfahren beim Senat eingegangen. 
Ausschöpfung des Rechtsweges durch den Betroffenen, weiterhin als letzte Instanz entscheiden.

\section{Das Amparo-Verfahren}

Ebenso wie beim Habeas Corpus ist auch die Möglichkeit der Einlegung des AmparoRechtsmittels in Art. 48 der Verfassung Costa Ricas zum Grundrecht erklärt worden. Die Kompetenz des Verfassungssenates ergibt sich aus dem Verweis auf Art. 10 der Verfassung. Die LJC unterteilt das Amparo-Verfahren im Titel III in Amparo gegen Behörden, Organe, Beamte und Angestellte der öffentlichen Hand ${ }^{38}$, Amparo gegen juristische oder natürliche Personen des privaten Rechts ${ }^{39}$ und Amparo zur Durchsetzung des Rechts auf Widerruf und Gegendarstellung ${ }^{40}$. Das Amparo-Verfahren findet in Lateinamerika seinen Ursprung im mexikanischen Rechtssystem und wurde in Costa Rica erstmals in der Verfassung von 1949 aufgenommen. Es handelt sich um ein summarisches Verfahren zum Schutz der Grundrechte. Rechtsschutzbereich des Amparo-Verfahrens sind alle nicht durch das Rechtsmittel des Habeas-Corpus geschützten Grundrechte ${ }^{41}$. Ebenfalls wie beim Habeas-Corpus-Verfahren sind die Formalitäten auf ein Minimum beschränkt worden. In der vor 1989 geltenden "Ley de Amparo" war zunächst die Erschöpfung des Rechtsweges vorgesehen. Diese Voraussetzung wurde in de LJC abgeschafft. Sollte jedoch zunächst der Verwaltungsrechtsweg gewählt worden sein, so wird die Einlegungsfrist des Art. 35 LJC gem. Art. 31 LJC unterbrochen. Das Rechtsmittel kann, mit Ausnahme des Verfahrens zur Durchsetzung des Rechts auf Widerruf und Gegendarstellung, von jedermann $^{42}$ schriftlich eingelegt werden. Dabei werden die Kosten für eine telegrafische Einreichung übernommen ${ }^{43}$.

\section{Amparo-Verfahren gegen Behörden, Organe, Beamte und Angestellte der öfentlichen Hand}

Dieses mit Abstand am häufigsten durchgeführte Verfahren, ist in den Art. 29 bis 56 LJC geregelt. Das Rechtsmittel kann, wie oben bereits erwähnt, von jedermann eingelegt werden. Passiv legitimiert ist derjenige Beamte oder Angestellte, der die Verletzungs-

38

39

40

41

42

43

Amparo contra órganos o servidores públicos.

Amparo contra sujetos de derecho privado.

Derecho de rectificación o respuesta.

Art. 29 I LJC: "El recurso de amparo garantiza los derechos y libertades fundamentales a que se refiere esta Ley, salvo los protegidos por el de hábeas corpus."

Art. 33 LJC: "Cualquier persona podrá interponer el recurso de amparo."

Art. 38 III LJC: "El recurso no está sujeto a otras formalidades ni requerirá autenticación. Podrá plantearse por memorial, telegrama u otro medio de comunicación que se manifieste por escrito, para lo cual gozará de franquicia telegráfica." 
handlung durchgeführt hat. Hat er auf Weisung eines Vorgesetzten gehandelt, so richtet sich das Rechtsmittel auch gegen ihn. Ist die handelnde Person unbekannt, so ist der Leiter der entsprechenden Behörde oder des entsprechenden Organs passiv legitimiert ${ }^{44}$. Zum Verfahren werden weiterhin die durch die angegriffene Regel oder den angegriffenen Verwaltungsakt Begünstigten hinzugezogen. Andere, die ein berechtigtes Interesse am Ausgang des Verfahrens haben, können einer der streitenden Parteien beitreten. Das Rechtsmittel kann bereits eingelegt werden, wenn die Verletzung von Grundrechten droht $^{45}$, solange sie andauert ${ }^{46}$ und bis zu zwei Monate nach Beendigung der direkten Auswirkungen auf den Betroffenen ${ }^{47}$.

Etwas unübersichtlich ist in der LJC die Behandlung der Amparoverfahren gegen die ein Gesetz vollziehenden Akte. Gem. Art. 30 Nr. a LJC ist das Amparoverfahren nicht statthaft gegen Gesetze und andere Normen, es sei denn, man greift gemeinsam mit den Gesetzen und Normen den Vollzugsakt an, der hierauf begründet ist. Weiterhin ist das Amparoverfahren gem. Art. $30 \mathrm{Nr}$. a LJC auch dann gegen Gesetze statthaft, wenn diese

44

Art. 34 I LJC: "El recurso se dirigirá contra el servidor o el titular del órgano que aparezca como presunto autor del agravio. Si uno u otro hubiese actuado en cumplimiento de órdenes o instrucciónes impartidas por un superior, o con su autorización o aprobación, se tendrá por establecido el amparo contra ambos, sin perjuicio de lo que se decida en sentencia. De ignorarse la identidad del servidor, el recurso se tendrá establecido contra el jerarca."

45

Laut $R$. Hernández Valle muß die Verletzung unmittelbar bevorstehen und es müssen hierfür konkrete Anzeichen vorliegen. $R$. Hernández Valle: "La tutela de los derechos fundamentales", Juricentro, 1990, S. 91.

46

Handelt es sich bei den angeblich verletzten Rechten um Eigentumsrechte oder um solche Rechte, auf die man wirksam verzichten kann, so bestimmt Art. 35 II LJC, daß das Rechtsmittel innerhalb der ersten zwei Monate nach Kenntnis der Verletzungshandlungen einzulegen ist. Wird die Frist nicht eingehalten, wird das Rechtsmittel gem. Art. 35 in Verbindung mit Art. 9 I LJC von Amts wegen als unzulässig zurückgewiesen. Da gem. Art. 35 I LJC das Amparoverfahren angestrengt werden kann, solange die Verletzungshandlung andauert, Art. 35 II LJC jedoch Ausnahmen vorsieht für die Verletzung von Rechten, auf die man wirksam verzichten kann, ist man gut beraten, wenn man die hier vorgesehene Zweimonatsfrist ab Kenntnisnahme der Verletzungshandlung einhält. Der Verfassungssenat hat nähmlich die Ausnahme des Art. 35 II LJC nicht nur auf die Fälle angewandt, in denen ausdrücklich auf das Recht verzichtet wurde, sondern ist nach Ablauf der Frist von zwei Monaten auch von einem konkludenten Verzicht ausgegangen (soweit ein Rechtsverzicht überhaupt möglich ist. Vgl. z.B. Urteile 80-90 vom 19.01.90; 1-91 vom 04.01.91; 605-91 vom 20.03.91). Dies erscheint insofern nicht unproblematisch, als somit die Ausnahme zur Regel gemacht wird (ebenso M. Murillo: "Plazo para interponer el recurso de amparo", in: Revista de Derecho Constitucional 2, May-August 1991, S.93 ff (95)). Diese sehr weite Auslegung des Senates erklärt sich wohl unter anderem aus der hohen Arbeitsbelastung und den Schwierigkeiten, ältere Sachverhalte aufzuklären. Sinnvoller wäre es meines Erachtens, die Voraussetzung der Ausschöpfung des Rechtsweges wieder einzuführen. Dies insbesondere deshalb, als dieser Rechtsweg gem. Art. 36 LJC auch nach der Zurückweisung des Amparo-Rechtsmittels wegen Verf ristung noch offen steht, soweit die dort vorgesehenen längeren Fristen nicht auch bereits abgelaufen sind.

47

Art. 35 I LJC: "El recurso de amparo podrá interponerse en cualquier tiempo mientras subsista la violación, amenaza, perturbación o restricción, y hasta dos meses después de que hayan cesado totalmente sus efectos directos respecto al perjudicado." 
irgendeinen Betroffenen ${ }^{48}$ (nicht notwendigerweise den Kläger) gegenwärtig und unmittelbar (also ohne die Notwendigkeit eines Vollzugsaktes) in seinen Grundrechten verletzen. Nachdem nun Art. 30 a LJC die Möglichkeit von Amparo-Verfahren gegen Gesetze und Normen eröffnet, ordnet Art. 48 I bzw. II LJC im Falle eines direkten oder indirekten Amparos gegen ein Gesetz oder eine Norm das Ruhen des Verfahrens an und verpflichtet entweder den Senat oder den Präsidenten des Senats, den Beschwerdeführer aufzufordern, eine "Acción de Inconstitucionalidad" innerhalb der nächsten 15 Werktage in die Wege zu leiten. Macht der Beschwerdeführer dies nicht, ruht das Verfahren ${ }^{49}$. In der Praxis kann der Beschwerdeführer jedoch auf eine Verbescheidung des Amparo-Verfahrens bestehen. Finden die mit einer Amparobeschwerde angegriffenen Akte eine begründete Stütze in einem Gesetz oder in einer Norm, egal ob diese mit angegriffen wurde oder nicht, hat der Senat den Beschwerdeführer gem. Art. 48 I LJC ebenso aufzufordern, eine "Acción de Inconstitucionalidad" anzustrengen, soweit er das Gesetz für verfassungswidrig hält. Eine Inzidentkontrolle ist nicht möglich. In der Praxis entscheidet der Senat nicht immer einheitlich, wann das Amparoverfahren ruhen soll und die Aufforderung zur Einlegung der Normenkontrollbeschwerde ergehen soll. Dies geschieht jedenfalls nicht, wenn das entsprechende Gesetz bereits auf seine Verfassungsmäßigkeit überprüft wurde. Sind diese Voraussetzungen nicht gegeben, werden die Gründe für eine direkte Entscheidung oder eine Aufforderung zur Einlegung der Normenkontrollbeschwerde nicht immer deutlich ${ }^{50}$. Art. 37 LJC ermöglicht insbesondere auch eine Entscheidung des Senats über den Vollzugsakt eines Gesetzes oder einer Norm, wenn der Beschwerdeführer die Frist zur Einlegung der Normenkontrollbeschwerde verstreichen läßt. Übersichtlicher und einfacher wäre es, wenn die direkte und unmittelbare Betroffenheit des Beschwerdeführers bei einem Amparo gegen Gesetze uneingeschränkt eingeführt würde und die Verfassungswidrigkeit der Gesetze und Normen, auf denen angegriffene Akte beruhen, inzident überprüft würden. So könnte man sich den Zwischenschritt mit der Aufforderung zur Einlegung der Normenkontrollbeschwerde sparen und müßte das Amparoverfahren nicht ruhen lassen, ohne daß dadurch eine Verkürzung des Rechtsschutzes erfolgt. Das Rechtsmittel muß lediglich schriftlich eingelegt werden und das angeblich verletzte Recht sowie die Verletzungshandlung umschrieben werden. Grundsätzlich nicht erforderlich ist die Benennung der verletzten Verfassungsnorm. Beruft sich der Beschwerdeführer jedoch auf eine Vorschrift aus einem internationalen Abkommen, so hat er diese konkret zu benennen ${ }^{51}$.

Art. 33 LJC: "Cualquier persona podrá interponer el recurso de amparo".

49

Das Gesetz bestimmt eine "Archivienung" der Akte.

50

Dementsprechend sind die Entscheidungen in der Materie auch häufig sehr kontrovers, vgl. z.B. Entscheidung 524-91 vom 08.03.91.

51

Die internationalen Menschenrechtsabkommen, denen Costa Rica beigetreten ist, sind neben der Verfassung Prüfungsmaßstab für den Senat. 
Ebenso wie beim Habeas-Corpus-Verfahren wird das Amparoverfahren zunächst von einem Richter bearbeitet, der die beklagte Seite (Behörde oder öffentlicher Angestellter bzw. Beamter) auffordert, innerhalb von einer Frist von bis zu drei Tagen in einem Bericht Stellung zur Amparo-Beschwerde zu nehmen. Der Bericht gilt gem. Art. 44 II LJC als unter Eid abgegeben und zieht bei bewußten Ungenauigkeiten oder Fehlern die entsprechenden strafrechtlichen Folgen nach sich. Der für die Erstellung des Berichts gewährte Zeitraum erscheint sehr kurz, insbesondere wenn man an die möglichen Folgen bei Fehlern und Ungenauigkeiten denkt. Bei der hohen Anzahl an Amparo-Verfahren ist ein nicht unerheblicher Teil der Verwaltung ständig blockiert mit der vordringlichen Erstellung entsprechend gewissenhafter Berichte. Wird in der vorgesehenen Frist überhaupt kein Bericht abgegeben, greift in der Regel die Wahrheitsvermutung zugunsten des Beschwerdeführers ein. Der Senat kann gem. Art. 45 LJC allerdings auch eigene Ermittlungen anstellen, soweit er diese für notwendig hält. Ein wichtiger Grund für die hohe Anzahl von Amparo-Verfahren vor dem Verfassungssenat ist die aufschiebende Wirkung des Rechtsmittels. Diese tritt automatisch ein und entfällt nur dann, wenn durch die Aufschiebung besonders schwerwiegende Folgen für die Allgemeinheit zu erwarten sind, die schwerer wiegen, als die dem Beschwerdeführer drohenden Schäden bei einer sofortigen Vollziehung. In diesem Fall kann der Senat auf Antrag der erlassenden Behörde oder auch von Amts wegen die aufschiebende Wirkung aufheben. Eine sofortige Vollziehung von mit dem Amparo-Rechtsmittel angegriffenen Verwaltungsakten ist deshalb in der Praxis nur in extremen Ausnahmefällen möglich. Dies bedeutet für den Beschwerdeführer in der Regel einen Zeitgewinn und behindert die Arbeit der öffentlichen Verwaltung in nicht zu rechtfertigender Weise. Denkbar wäre hier eine Auflistung von Ausnahmen für den automatischen Eintritt der aufschiebenden Wirkung ähnlich der Auflistung in § 80 II VwGO. Für den Eintritt der aufschiebenden Wirkung könnte man auch auf die Erfolgsaussichten des Rechtsmittels abstellen. Zudem könnte die Entscheidung über die Aufhebung der aufschiebenden Wirkung vom den Fall bearbeitenden Einzelrichter getroffen werden, um den Senat zu entlasten. Auch hier erscheint es trotz allem am sinnvollsten, den Beschwerdeführer zunächst auf den Verwaltungsrechtsweg zu verweisen, um bereits auf diesem Wege Abhilfe zu suchen und damit die Entscheidungen über die Aufhebung der aufschiebenden Wirkung den Verwaltungsgerichten zu überlassen. Eine Neuigkeit im verfassungsrechtlichen Amparo-Verfahren ist gem. Art. 41 IV LJC die Möglichkeit des Erlasses vorläufiger Maßnahmen durch den bearbeitenden Richter oder den Präsidenten des Verfassungssenates zur Verfahrenssicherung und zur Abwendung möglicher Schäden.

Im Falle der Zulässigkeit und Begründetheit des Amparo-Rechtsmittels wird die beklagte Partei aufgefordert, den Beschwerdeführer in seinen vollen Rechten wieder herzustellen und den entstandenen Schaden zu ersetzen. Die Vollstreckung des Schadensersatzes erfolgt im Wege des Verwaltungsvollstreckungsrechts. Zur Durchsetzung der Urteile kann der Verfassungssenat gem. Art. 53 LJC die Durchführung von Disziplinarmaßnahmen gegen die die Anordnungen nicht erfüllenden Angestellten und Beamten anordnen. Laut 
Angaben der Verfassungsrichter werden die Anordnungen in der Regel sofort befolgt, ohne daß auf diese Maßnahmen zurückgegriffen werden muß. Ist die Beschwerde unzulässig, unbegründet oder wird sie zurückgenommen, so kann der Senat dem Beschwerdeführer gem. Art. 51 III LJC die entstandenen Kosten des Verfahrens in Form einer Mißbrauchsgebühr auferlegen.

\section{Amparo gegen juristische und natürliche Personen des privaten Rechts}

Gemäß Art. 57 bis 65 LJC kann man mit der Amparo-Beschwerde auch gegen Private vorgehen, soweit diese im öffentlichen Auftrag handeln oder aus rechtlichen oder tatsächlichen Gründen in einem Überordnungsverhältnis zum Beschwerdeführer stehen und wenn andere Rechtsmittel keinen rechtzeitigen und ausreichenden Schutz der Grundrechte bieten $^{52}$. Ursprünglich sind die Grundrechte als Abwehrrechte gegen den Staat konzipiert worden. Dementsprechend richtete sich das mexikanische Amparoverfahren, Vorbild aller lateinamerikanischen Amparoverfahren, nur gegen die "Autorität", womit staatliche Behörden gemeint waren ${ }^{53}$. Durch die relative Wirksamkeit und den schnellen Rechtsschutz, den das Amparoverfahren zum Schutz der Grundrechte bietet, wurde bald die Tendenz erkennbar, dieses auch auf Verfahren gegen Private auszudehnen. Damit wurde implizit die Drittwirkung der Grundrechte bejaht, ohne daß jedoch eine intensivere wissenschaftliche Auseinandersetzung zu diesem Punkt stattfand. Dabei ist die Frage der Drittwirkung von Grundrechten nicht unproblematisch und war auch z.B. in der deutschen Verfassungsdoktrin stark umstritten ${ }^{54}$. Eine ausdrücklich von der Verfassung vorgesehene Drittwirkung kann man in der Koalitionsfreiheit des Art. 25 der costa-ricanischen Verfassung erkennen. Satz 2 dieses Artikels bestimmt, daß niemand gezwungen werden kann, Mitglied in einer Vereinigung zu werden ${ }^{55}$. Die negative Koalitionsf reiheit hat somit eine in der Verfassung ausdrücklich bestimmte Drittwirkung. Die Frage der Drittwirkung von Grundrechten wird in Lateinamerika in erster Linie aus verfahrensrechtlicher Sicht behandelt. Art. 25 des Interamerikanischen Menschenrechtsabkommens von San José

Art. 57 LJC: "El recurso de amparo también se concederá contra la acciones u omisiones de sujetos de Derecho Privado, cuando éstos actúen o deban actuar en ejercicio de funciones o potestades públicas, o, se encuentren, de derecho o de hecho, en una posición de poder frente a la cual los remedios jurisdiccionales comunes resulten claramente insuficientes o tardíos para garantizar los derechos o libertades fundamentales a que se refiere el artículo 2, inciso a) de esta Ley."

H. Fix Zamudio: "Latinoamérica: Constitución, Proceso y Derechos Humanos, Mexico D.F., 1988, S. 120.

Vgl. für alle: A. Bleckmann: " Staatsrecht II - Die Grundrechte", Köln, Berlin, Bonn, München, 3. Auflage 1989, S. $175 \mathrm{ff}$.

Art. 25 Constitución Política de Costa Rica: "Los habitantes de la República tienen derecho de asociarse para fines lícitos. Nadie podrá ser obligado a formar parte de asociación alguna." 
(Amerikanische Menschenrechtskonvention von 1969) ${ }^{56}$ bestimmt, daß der Verletzte bei einer Einschränkung der in der Verfassung, den Gesetzen oder dem Abkommen festgelegten Rechte und Freiheiten das Recht hat, eine wirksame Beschwerde bei einer nationalen Instanz einzulegen, selbst wenn die Verletzung von Personen begangen worden ist, die in offizieller Eigenschaft gehandelt haben ${ }^{57}$. Hieraus zieht z.B. H. Fix Zamudio die Konsequenz, daß das Amparoverfahren auch gegen Private statthaft sein müsse ${ }^{58}$. Aus den staatlichen Schutzpflichten ließe sich demnach die Drittwirkung von Grundrechten herleiten. Erstmals in Lateinamerika scheint sich der Oberste Gerichtshof Argentiniens mit der Frage der Drittwirkung von Grundrechten, allerdings auch im Rahmen der Statthaftigkeitsprüfung eines Amparo-Verfahrens, befaßt zu haben. Im Fall "Samuel Kot" ${ }^{59}$ entschied der Gerichtshof, daß die in der Verfassung festgeschriebenen Grundrechte gegen Jedermann (erga omnes) durchsetzbar seien. Diese Rechtsprechung wurde von verschiedenen Gesetzgebern, zunächst in Argentinien, später auch in anderen lateinamerikanischen Ländern, aufgegriffen und gesetzlich geregelt. Die direkte Drittwirkung der Grundrechte ergibt sich in Costa Rica aus den Art. 57 ff LJC. Die Beschwerde kann von Jedermann erhoben werden. Passiv legitimiert ist gem. Art. 59 derjenige, der die angebliche Verletzungshandlung vorgenommen hat, bei juristischen Personen der entsprechende Vertreter. Für die Einlegung gilt die in Art. 35 LJC geregelte Frist. Die Zurückweisung der Beschwerde hat keine präjudizierende Wirkung auf ein späteres Straf- oder Zivilverfahren. Im übrigen verweist Art. 65 LJC auf die Vorschriften des Amparo gegen Behörden, Organe, Beamten und Angestellte des öffentlichen Dienstes. Bei den Amparo-Verfahren gegen Private handelt es sich fast ausschließlich um arbeitsrechtliche Fragen. Hier macht sich der mangelnde Rechtsschutz, den das veraltete Arbeitsgesetz bietet, negativ bemerkbar. Eine Entlastung des Verfassungssenates könnte durch die Einführung eines summarischen Verfahrens vor den Arbeitsgerichten (Amparo laboral) erreicht werden.

Pacto Interamericano de Derechos Humanos, suscrito en Costa Rica en noviembre de 1969, Art. 25 : "Toda persona tiene derecho a un recurso sencillo y rápido o a cualquier otro recurso efectivo ante los jueces o tribunales competentes que ampare contra actos que violen sus derechos fundamentales reconocidos por la Constitución, la ley o la presente Convención, aun cuando tal violación sea cometida por personas que actúen en ejercicio de sus funciones oficiales....".

Die Vorschrift entspricht ziemlich genau dem Wortlaut des Art. 13 EMRK wonach der Verletzte, bei einer Einschränkung seiner Rechte und Freiheiten, das Recht hat, eine wirksame Beschwerde bei einer nationalen Instanz einzulegen, selbst wenn die Verletzung von Personen begangen worden ist, die in offizieller Eigenschaft gehandelt haben. Nach diesem Wortlaut muß dem Verletzten dieses Recht um so eher zustehen, wenn die Privaten in privater Eigenschaft gehandelt haben. Trotzdem ist die Drittwirkung der EMRK umstritten, vgl. z.B. A. Bleckmann, "Europarecht, Das Recht der Europäischen Wirtschaftsgemeinschaft", 4. Auflage 1984, § 4 XI. H. Fix Zamudio: (Fn. 53), S. 121.

Entscheidung des Obersten Gerichtshofs in Buenos Aires vom 5. September 1958. Die Fabrik vom Kläger Samuel Kot war von einer Gruppe Arbeiter besetzt worden. 


\section{Recht auf Erwiderung und Berichtigung}

Um die Pflichten, die mit dem Recht auf Meinungs- und Äußerungsfreiheit (Artt. 28 und 29 der Verfassung Costa Ricas) verbunden sind, wirksam durchzusetzen, sieht die LJC in ihren Artt. 66 bis 70 ein besonderes Amparo-Verfahren vor. Hiermit wird auch der Verpflichtung aus Art. 14 der Amerikanischen Menschenrechtskonvention Rechnung getragen, wonach das Recht auf Erwiderung und Berichtigung ebenfalls garantiert wird ${ }^{60}$. Das einzuhaltende Verfahren wird in Art. 69 der LJC ausführlich beschrieben. Der Beschwerdeführer hat den Text der Erwiderung oder Berichtigung innerhalb von fün Tagen ab Veröffentlichung der angegangenen Äußerung zu fertigen und diesen zur Publikation an das entsprechende Kommunikationsmittel weiterzureichen. Soweit es sich um ein täglich erscheinendes Publikationsmittel handelt (Tageszeitung), ist die Erwiderung innerhalb von drei Tagen nach Abgabe zu veröffentlichen, ansonsten in der nächsten Ausgabe. Die Veröffentlichung hat in gleicher Weise zu erfolgen wie die des angegriffenen Textes. Die Erwiderung muß sich in einem angemessenen Rahmen halten und darf keine Kommentare enthalten, die nicht in direkter Beziehung zur angegriffenen Veröffentlichung stehen. Weigert sich der Inhaber des Publikationsmittels, die Erwiderung zu veröffentlichen, ist die Amparo-Beschwerde nach Art. 66 LJC statthaft. Die beklagte Seite wird innerhalb von 24 Stunden gehört, und die Entscheidung des Senats erfolgt in einer Frist von drei Tagen nach der Anhörung. Vollstreckt wird die Entscheidung des Verfassungssenates auf dem Zivilrechtswege. Hier stellt sich die Frage, warum nicht auch zunächst ein Zivilgericht mit der Frage der Erwiderung und Berichtigung befaßt wird. Die Anzahl dieser speziellen Amparo-Verfahrens ist sehr niedrig und stellt keine außergewöhnliche Belastung für den Senat dar. Die letzte, zum Zeitpunkt der Fertigstellung dieser Arbeit veröffentlichte Entscheidung, stammt aus dem Jahre $1990^{61}$.

Das Amparo-Verfahren hat sich in Costa Rica zu einem Parallelrecht entwickelt, da verwaltungsrechtliche und auch arbeitsrechtliche Streitigkeiten (Amparo gegen Dritte) so wesentlich schneller beschieden werden, als in den dafür eigentlich vorgesehenen Rechtswegen. Dies wird dadurch begünstigt, daß der Senat sehr großzügig das Vorliegen einer verfassungsrechtlichen Streitigkeit feststellt und letzlich in jedem Rechtsstreit auch einen Verfassungsstreit erkennt, soweit er über den Fall entscheiden will (zuletzt immer öfter in arbeitsrechtlichen Streitigkeiten). Entlastung für den Senat kann auch hier nur

60

Art. 14 I AMRK: "Jeder, der durch eine unrichtige oder beleidigende Behauptung oder Meinungsäußerung verletzt ist, die allgemein in der Öffentlichkeit durch ein gesetzlich geregeltes Kommunikationsmittel verbreitet wurde, hat unter den gesetzlich vorgesehenen Bedingungen das Recht auf 61 Erwiderung oder Berichtigung unter Benutzung desselben Kommunikationsweges."

Entscheidung des Senates Nr. 1292-90 vom 17. Oktober 1990. Das Rechtsmittel wurde als unstatthaft zurückgewiesen, da der Beschwerdeführer auf dem Weg des Amparo gegen Dritte eine Stellungnahme des Senates zu einer Aussage der Ärztekammer herbeiführen, nicht jedoch die Publikation einer eigenen Erwidenung durchsetzen wollte. 
eine Pflicht zur Rechtswegerschöpfung bringen, begleitet durch eine Reform des Verwaltungs- und des Arbeitsrechts (etwa durch die Einführung summarischer Verfahren in beiden Bereichen). Aufgrund der hohen Anzahl an Verfahren, für die der Senat erste und letzte Instanz und damit auch Tatsacheninstanz ist, kann eine gründliche Prüfung der einzelnen Fälle in all ihren Aspekten nicht immer erfolgen. Auch bei der Durchsicht der veröffentlichten Urteile stellt man fest, daß eine intensive wissenschaftliche Auseinandersetzung mit den aufgetauchten Problemen zum Teil zu kurz kommt. Hier wäre weniger mehr. Der Verfassungssenat wird als schnelle und endgültige Instanz "mißbraucht" und kann so seiner eigentlichen Aufgabe, die Verfassung allgemeinverbindlich zu interpretieren, nur noch mit großer Mühe nachkommen.

\section{Die Frage der Verfassungsmäßigkeit von Normen und Einzelakten}

Die Prüfung der Verfassungsmäßigkeit von Normen und Einzelakten, die nicht durch das Habeas-Corpus-Verfahren oder das Amparo-Verfahren gedeckt sind, ist nach den verschiedenen, im Titel IV der LJC vorgesehenen Verfahren, möglich. Dabei ist zunächst zu unterscheiden zwischen der vorbeugenden Normenkontrolle und der nachträglichen Kontrolle von Normen und Einzelakten sowie der Richtervorlage. Mit dem Ziel, einen lückenlosen Verfassungsschutz einzuführen, sieht die LJC drei unterschiedliche Formen der Normenkontrolle vor. Die vorbeugende Normenkontrolle lehnt sich an das französische Vorbild des "Conseil constitutionnel" an ${ }^{62}$. In Deutschland ist die vorbeugende Normenkontrolle, mit Ausnahme der Prüfung von völkerrechtlichen Verträgen, unbekannt. Bei Vertragsgesetzen nach Art. 59 II GG ist die Normenkontrolle schon dann zulässig, wenn das Gesetzgebungsverfahren bis auf die Ausfertigung des Vertragsgesetzes durch den Bundespräsidenten und die Verkündung abgeschlossen ist ${ }^{63}$. Eine Konsultationspflicht der Legislative ist ansonsten nicht vorgesehen. Die Verfassungsbeschwerde gegen Gesetze und die Richtervorlage sind, mit einigen Abweichungen, im deutschen Verfassungsrecht bekannt.

\section{Die vorbeugende Normenkontrolle}

Kapitel II, Art. 96 bis 101 LJC regelt die vorbeugende Normenkontrolle (consulta de constitucionalidad). Es handelt sich dabei um Anfragen an den Verfassungssenat mit der Bitte um Stellungnahme zur Verfassungsmäßigkeit von Reformvorhaben, Gesetzesprojekten, Verwaltungsverträgen, der Geschäfts- und Disziplinarordnung des Kongresses und von völkerrechtlichen Verträgen. Bei Reformvorhaben zur Verfassung und zur LJC

Als Vorbild dürften auch die Regelungen in Portugal gedient haben. Zu Einzelheiten zur vorbeugenden Normenkontrolle siehe Franck Moderne: "El control previo de constitucionalidad en la Europa contemporánea", in: La Jurisdicción Constitucional, (Fn. ), S. 149 ff.

BVerfGE 1, S. 413; 2, S. 169. 
besteht gem. Art. 96 Nr. a LJC eine Konsultationspflicht ${ }^{64}$. Die entsprechende Anfrage ist vom Direktorium der gesetzgebenden Versammlung zu stellen. Ansonsten ist die Konsultation fakultativ und muß von der zuständigen Stelle (Oberster Gerichtshof, Oberstes Wahlgericht, Rechnungshof, soweit sie betroffen sind und vom Ombudsmann für Menschenrechte) oder Abgeordnetengruppe (mindestens zehn Abgeordnete) beantragt werden. Die Konsultationen sind schriftlich vorzulegen, wobei die fakultativen Anfragen mit den Angaben der angeblichen Verfassungsverletzungen begründet werden müssen. Die Frist für die Stellungnahme beträgt laut Art. 101 I LJC einen Monat. Die Stellungnahme ist nur dann verbindlich für die Legislative, wenn der Verfassungssenat eine formelle Verfassungswidrigkeit rügt. Gem. Art. 101 III LJC schließt die vorbeugende Verfassungskontrolle eine spätere, nachträgliche Verfassungskontrolle nicht aus. Diese ist dann in jedem Fall verbindlich. Die Konsultation hat in Costa Rica eine durchweg positive Wirkung gehabt. Anfängliche Bedenken, dies könne zu einer "Richterregierung" führen, haben sich nicht realisiert. Statt dessen kann man bei der Legislative eine stärkere Beachtung der Verfassung erkennen. Auf diese Weise hat sie selber erheblich an Ansehen gewonnen.

\section{Die nachträgliche Kontrolle von Normen und Einzelakten}

Art. 73 in Kapitel I, Titel IV der LJC bestimmt in seinen Nummern a), b), e) und f) den Prüfungsgegenstand der "Acción de Inconstitucionalidad", die mit der deutschen Verfassungsbeschwerde nach Art. 93 I Nr. 4 a GG vergleichbar ist. (Gemeinsam decken das "Habeas-Corpus-Verfahren", das "Amparo-Verfahren" und die "Acción de Inconstitucionalidad" den Schutzbereich der deutschen Verfassungsbeschwerde nach Art. 93 I Nr. 4 a GG ab). Die Ziffern c), ch) und d) des Art. 73 LJC stellen weniger den Prüfungsgegenstand als den Prüfungsmaßstab dar ${ }^{65}$. Die "Acción de Inconstitucionalidad" ist gem.

64

Art. 96 LJC: "Por la vía de consulta de constitucionalidad, la jurisdicción constitucional ejercerá la opinión consultiva previa sobre los proyectos legislativos, en los siguientes supuestos:

a) Preceptivamente, cuando se trate de proyectos de reformas constitucionales, o de reformas a la presente Ley, así como de los tendientes a la aprobación de convenios o tratados internacionales,

65 inclusive las reservas hechas o propuestas a unos u otros."

Art. 73 LJC: "Cabrá la acción de inconstitucionalidad:

a) Contra las leyes y otras disposiciones generales, incluso las originadas en actos de sujetos privados, que inf rinjan, por acción u omisión, alguna norma o principio constitucional.

b) Contra los actos subjetivos de las autoridades públicas, cuando infrinjan, por acción u omisión, alguna norma o principio constitucional, si no fueren susceptibles de los recursos de hábeas corpus o de amparo.

c) Cuando en la formación de las leyes o acuerdos legislativos se viole algún requisito o trámite sustancial previsto en la Constitucición o, en su caso, establecido en el Reglamento de Orden, Dirección y Disciplina Interior de la Asamblea Legislativa.

ch) Cuando se apruebe una reforma constitucional con violación de normas constitucionales de procedimiento.

d) Cuando alguna ley o disposición general infrinja el artículo $7^{\circ}$, párrafo primero, de la Constitución, por oponerse a un tratado público o convenio internacional. 
Art. 73 Nr. a LJC auch gegen von Privatsubjekten erlassene "allgemeine Regelungen" statthaft. In der Praxis dürften hiermit z.B. Benutzerordnungen von Privateinrichtungen gemeint sein. Nicht darunter fallen mit Sicherheit allgemeine Geschäftsbedingungen. Rechtsprechung zu dieser Frage wurde jedoch bisher noch nicht veröffentlicht. Bei der Durchsicht der Prüfungsgegenstände wird man erkennen, da $\beta$ auch hier der Gesetzgeber bemüht war, einen möglichst umfangreichen Rechtsschutz zu bieten und keine Lücke entstehen zu lassen. Nur so läßt sich z.B. die Regelung des Art. 73 Nr. b LJC erklären, der alle nicht mit dem Habeas-Corpus-Verfahren oder dem Amparo-Verfahren angreifbaren Einzelakte unter den Schutz der "Acción de Inconstitucionalidad" stellt. Auch hierzu ist bisher noch keine Rechtsprechung ergangen ${ }^{66}$. Mit der "Acción de Inconstitucionalidad" kann man sich auch gegen ein Unterlassen der öffentlichen Gewalt wehren, wie Art. 73 Nr.f LJC bestimmt ${ }^{67}$. Nicht statthaft dagegen ist das Rechtsmittel gegen Gerichtsentscheidungen und Beschlüsse sowie gegen Entscheidungen des Obersten Wahlgerichts im Zusammenhang mit seiner Funktion. Die "Acción de Inconstitucionalidad" kann von jedermann erhoben werden. Vorraussetzung ist gem. Art. 75 I LJC jedoch, daß die Beschwerde aus einem anhängigen Verfahren heraus erhoben wird und angemessen ist, um das angegriffene Recht zu schützen ${ }^{68}$. Als "anhängiges Verfahren" im Sinne des Art. 75 I LJC gilt auch das Widerspruchsverfahren, welches im costa-ricanischen Verwaltungsrecht bekannt ist und ähnlich dem deutschen Widerspruchsverfahren gestaltet ist. Der Senat hat in seiner Rechtsprechung wiederholt darauf hingewiesen, was er unter "angemessen" versteht. Demnach muß die "Acción de Inconstitucionalidad" zu dem gegebenen Zeitpunkt absolut notwendig sein, um das betroffene Recht zu schützen ${ }^{69}$. Bedenkt man, daß durch die "Acción de Inconstitucionalidad" das anhängige Verfahren unterbrochen wird, ist diese Auslegung des Wortes "angemessen" nur konsequent. Ansonsten würde nämlich der Eintritt des Rechtsschutzes durch die Verbescheidung des anhängigen Verfahrens sinnlos verzögert. Hier liegt aber auch eine Gefahr. Prinzipiell kann jeder

e) Cuando en la suscripción, aprobación o ratificación de los convenios o tratados internacionales, o en su contenido o efectos se haya inf ringido una norma o principio constitucional o, en su caso, del Reglamento de Orden, Dirección y Disciplina Interior de la Asamblea Legislativa. En este evento, la declaratoria se hará solamente para los efectos de que se interpreten y apliquen en armonía con la constitución o, si su contradicción con ella resultare insalvable, se ordene su desaplicación con efectos generales y se proceda a su denuncia. f) Contra la inercia, las omisiones y las abstenciones de las autoridades públicas.

Mit Ausnahme der Entscheidung 1604-90 vom 09. November 1990, in der jedoch die Anwendbarkeit des Art. 73 Nr. b LJC auf den konkreten Fall verneint wurde. Interessant hierzu ist das Urteil des Senates 1463-90 vom 30.10.90.

Art. 75 I LJC: "Para interponer la acción de inconstitucionalidad es necesario que exista un asunto pendiente de resolver ante los tribunales, inclusive de hábeas corpus o de amparo, o en el procedimiento para agotar la vía administrativa, en que se invoque esa inconstitucionalidad como medio razonable de amparar el derecho o interés que se considera lesionado."

Entscheidung Nr. 305-90 vom 22. 03.1990: "...ni aún es suficiente que la acción de inconstitucionalidad sea un medio de amparar el derecho o interés que se considera lesionado; es preciso que el medio sea razonable, es decir, absolutamente necesario en ese preciso momento." 
anhängige Rechtsstreit durch die Einlegung einer "Acción de Inconstitucionalidad" verzögert werden. Der Senat muß in jedem Fall erst prüfen, ob es auf die angegriffene Rechtsnorm im besagten Streit überhaupt ankommt. Hierzu muß er aber eine formell- und materiellrechtliche Prüfung des anhängigen Falles durchführen. Dies kann nicht Aufgabe eines bereits überlasteten Verfassungssenates sein und dürfte auch als Einmischung in die Jurisdiktion der anderen Richter empfunden werden. Um eine wiederholte Verzögerung von anhängigen Verfahren zu vermeiden, beschränkt Art. 76 LJC die "Acciones de Inconstitucionalidad" auf höchstens eine pro Verfahren. Eine "Acción de Inconstitucionalidad" nach Art. 73 Nr. a LJC wäre dann überflüssig, wenn man beim Amparo-Verfahren die Inzidentkontrolle der Normen, auf die die angegriffenen Akte beruhen, durchführen würde nachdem zuvor der Rechtsweg erschöpft worden ist. Dabei sollte man auch nicht vergessen, daß die LJC die Richtervorlage vorsieht. Das Verfahren nach Art. 73 Nr. a LJC ist eine Art zusätzliche Möglichkeit der "Parteivorlage". Meines Erachtens wird hiermit unnötig in die Kompetenzen des Richters eingegriffen und den Parteien eine überflüssige Möglichkeit gegeben, den Rechtsstreit zu verzögern.

Eine Überprüfung außerhalb eines anhängigen Verfahrens ist dann möglich, wenn eine direkte und individuelle Betroffenheit des Beschwerdeführers nicht denkbar ist, wenn es um den Schutz diffuser Interessen geht oder solcher Interessen, die die Allgemeinheit als ganzes betreffen. Dabei reicht es allerdings nicht aus, daß eine rechtswidrige Norm schon aufgrund der Rechtswidrigkeit alleine gegen das Interesse der Gemeinschaft verstößt ${ }^{70}$. Dies würde zur Zulässigkeit der Popularklage führen, die der Gesetzgeber gerade nicht wollte. Weiterhin kennt die LJC noch die sogenannte "institutionelle Klagebefugnis" des Art. 75 III LJC. Demnach können der Präsident der Obersten Rechnungsprüfungsbehörde, der Generalanwalt, der Generalstaatsanwalt und der Ombudsmann die "Acción de Inconstitucionalidad" anstrengen, ohne daß ein anhängiges Verfahren nötig wäre.

Für die Einlegung der Beschwerde ist keine Frist vorgesehen, allerdings muß sie im Falle des Art. $73 \mathrm{Nr}$. a LJC erhoben werden, bevor eine rechtskräftigen Enscheidung ergangen ist. Dies ergibt sich zwar schon aus dem Wort "anhängig" in Art. 73 Nr. a LJC, wird jedoch in Art. 77 LJC noch einmal ausdrücklich festgehalten. Die Beschwerde ist schriftlich vorzulegen und zu begründen. Dabei sind die angeblich verletzten Normen und Prinzipien konkret zu zitieren. Sie bedarf der Beglaubigung. Beizufügen sind gem. Art. 79 I und II LJC der Schriftsatz des anhängigen Verfahrens und sieben unterschriebene Kopien der Beschwerde für die Richter des Verfassungssenates sowie weitere Kopien für die Generalanwaltschaft (Procuraduría) und die gegnerische Partei im anhängigen Verfahren.

70

Ständige Rechtsprechung des Verfassungssenates, siehe z.B. Entscheidungen 234-90 vom 28.02. 1990; 248-90 vom 07.03.1990; 495-90 vom 15.05.1990 und 680-90 vom 19.06.1990. Siehe hierzu auch $R$. Saborío Valverde: "La Admisibilidad den la Acción de Inconstitucionalidad", in: Revista de Derecho Constitucional, Mayo-Agosto 1991, San José, S. 35 ff (47/48). 
Die formellen Zulässigkeitsvoraussetzungen der "Acción de Inconstitucionalidad" werden zunächst vom Präsidenten des Verfassungssenates überprüft. Werden sie nicht erfüllt, weist er den Beschwerdeführer auf die Mängel hin und stellt ihm gem. Art. 80 I LJC eine Frist von drei Tagen zur Ergänzung bzw. Nachbesserung. Werden diese Handlungen vom Beschwerdeführer nicht in der vorgesehenen Frist durchgeführt, wird die Beschwerde vom Präsidenten des Senates zurückgewiesen. Gegen diese Zurückweisung kann innerhalb von weiteren drei Tagen ein Rechtsmittel eingelegt werden. Gem. Art. 80 II LJC hat dann der Senat über die Zurückweisung oder die Annahme der ursprünglichen Beschwerde zu entscheiden. Wird die Beschwerde durch den Präsidenten, oder nach Einlegung des Rechtsmittels durch den Senat, angenommen, ergeht eine Anordnung an die zuständige Behörde oder das zuständige Gericht des anhängigen Verfahrens mit dem Inhalt, dieses nicht zu verbescheiden. Bis auf die Entscheidung können allerdings weitere Prozeßhandlungen durchgeführt werden (z.B. Beweisaufnahmen). Die Anordnung ist im Amtsblatt (Boletín Oficial) dreimal hintereinander zu veröffentlichen. Innerhalb von fünfzehn Tagen nach der Veröffentlichung im Amtsblatt können diejenigen, die ein berechtigtes Interesse daran haben, zu der "Acción de Inconstitucionalidad" Stellung nehmen. Nach Ablauf der Frist wird ein Termin für eine mündliche Verhandlung, wie in Art. 10 LJC vorgesehen, festgesetzt. Die Entscheidung des Verfassungssenates hat innerhalb des ersten Monats nach der mündlichen Verhandlung zu ergehen. Wird die Beschwerde zurückgewiesen, ist auf alle Punkte der Begründung der "Acción de Inconstitucionalidad" einzugehen. Eine ablehnende Entscheidung hat, wie Art. 87 II LJC bestimmt, nur Auswirkungen inter pares und schließt keine weiteren Beschwerden gleichen Inhalts aus anderen Verfahren heraus aus. Die zulässige und begründete Beschwerde führt dagegen zur Erklärung der Verfassungwidrigkeit der angegriffenen Norm, Handlung oder Unterlassung. Sie wirkt erga omnes. Grundsätzlich ist diese Entscheidung auch rückwirkend, die Rückwirkung kann jedoch vom Senat eingeschränkt werden, um Sicherheit, Gerechtigkeit und sozialen Frieden zu gewähren und zu sichern. Sind Straf- und Disziplinarverfahren betroffen, so tritt eine Rückwirkung gem. Art. 92 LJC uneingeschränkt zugunsten der Betroffenen ein.

\section{Die Richtervorlage}

Kapitel III, Artt. 102 bis 108 LJC regelt die Richtervorlage. Gem. Art. 102 I LJC kann jeder Richter, soweit er Zweifel über die Verfassungsmäßigkeit einer von ihm in einem Rechtsstreit anzuwendenden Norm oder zu beurteilenden Handlung oder Unterlassung hat, diese dem Verfassungssenat zur Entscheidung vorlegen. Ob der Richter vorlegt, liegt nach dieser Vorschrift in seinem Ermessen. Art. 8 Abs. 1 des neuen Gerichtsordnungsgesetzes verpflichtet den Richter jedenfalls dann zur Vorlage, wenn er Zweifel an der Verfassungsmäßigkeit einer Norm hat (je nach Interpretation des Art 8 des Gerichtsorganisationsgesetzes könnte er eventuell dann selber entscheiden, wenn er von der Verfassungswidrigkeit überzeugt ist). Eine Vorlagepflicht besteht gem. Art. 102 II LJC in jedem Fall, wenn ein Wiederaufnahmeverfahren, wie von Art. 42 der Verfassung Costa Rica's 
vorgesehen, durchgeführt wird ${ }^{71}$. Ein rechtskräftig abgeschlossenes Straf verfahren kann dann wiederaufgenommen werden, wenn die Verletzung der Prinzipien des fairen Verfahrens, des rechtlichen Gehörs oder der fairen Verteidigung geltend gemacht wird. Dies bedeutet letztendlich, daß keine neuen Tatsachen für eine Wiederaufnahme des Verfahrens vorliegen müssen. Es handelt sich daher um eine Art "zweite Revision", diesmal durch den Verfassungsenat. Der Prüfungsrahmen des Senates ist dabei beschränkt auf die allgemeine Definition und Auslegung der angeblich verletzten Prinzipien und Rechte. Ausgangspunkt ist allerdings der konkrete Fall ${ }^{72}$.

Die Richtervorlage hat schriftlich zu erfolgen und ist ausführlich zu begründen. Ihr ist auch die Akte des anhängigen Verfahrens beizufügen, welches bis zur Entscheidung des Senates unterbrochen wird. Wird eine Vorlage nach Art. 102 I der LJC eingereicht, gibt der Senat den betroffenen Parteien Gelegenheit, wegen der gleichen Sache eine "Acción de Inconstitucionalidad" einzulegen. Dann wird nicht die Vorlage verbeschieden sondern das Verfahren der "Acción de Inconstitucionalidad" durchgeführt. Dies hat für die Parteien nur den Vorteil, daß sie sich im Rahmen einer mündlichen Verhandlung äußern und damit rechtliches Gehör verschaffen können. Kommt es zu dieser "Konvertierung", ist eine weitere "Acción de Inconstitucionalidad" ausgeschlossen, nicht aber im Falle der Richtervorlage. Die Richtervorlage ist innerhalb einer Frist von einem Monat zu beantworten ab Eingang beim Senat. Dabei kann der Senat sich auf einen Verweis auf seine Rechtsprechung beschränken, wenn er dies für ausreichend hält. Die Entscheidung des Senats hat gem. Art. 107 die gleichen Wirkungen wie die Entscheidung bei der "Acción de Inconstitucionalidad" und ist in gleicher Weise zu veröffentlichen. Die Richtervorlagen sind recht häufig, insbesondere im Bereich des Strafverfahrensrechts. Dies zeigt ein gesteigertes "Verfassungsbewußtsein" der Richter. Tatsächlich ist die Einflußnahme des Verfassungssenats auf die übliche Gerichtsbarkeit nicht zu unterschätzen. Es ist ihm gelungen, das bereits erwähnte "Verfassungsbewußtsein" zu wecken und eine verfassungskonforme Auslegung der Gesetze zu fördern. Nicht einsehbar ist allerdings, warum der "Parteivorlage" vor der Richtervorlage der Vorrang gegeben wird. Dies führt zu weiteren Verzögerungen und zusätzlichen Schriftwechseln sowie zu einer praktischen Verlagerung des Rechtsstreits vor den Verfassungssenat, der, wie bereits erwähnt, auch eine materielle Prüfung durchführen muß.

71

Art 42 der Verfassung Costa Rica's: "Un mismo juez no puede serlo en diversas instancias para la decisión de un mismo punto. Nadie podrá ser juzgado más de una vez por el mismo hecho punible.

Se prohibe reabrir cuasas penales fenecidas y juicios fallados con autoridad de cosa juzgada, salvo cuando proceda el recurso de revisión."

72

Siehe z.B. Entscheidung 3165-93 vom 06.07.1993. Die Prüfung, ob die Tatsachen, die angeblich die Rechte und Prinzipien verletzt haben, vorliegen, obliegt dem vorlegenden Gericht. 


\section{Die Lösung von Kompetenzkonflikten}

Der Verfassungssenat ist auch zuständig für die Entscheidung von Kompetenzstreitigkeiten zwischen den obersten staatlichen Organen und auch zwischen diesen und juristischen Personen des öffentlichen Rechts ${ }^{73}$. Diese Zuständigkeiten sind im Titel V und dort im einzigen Kapitel in den Artt. 109 bis 111 der LJC geregelt. Die Kompetenzstreitigkeit muß durch den entsprechenden Behörden- oder Institutionsleiter schriftlich vorgelegt werden. Der Präsident des Verfassungssenates gibt daraufhin dem Leiter der anderen Institution Gelegenheit zur Stellungnahme innerhalb von acht Tagen. Nach Ablauf der Frist entscheidet der Senat innerhalb von zehn Tagen. Die Frist wird nur dann verlängert, wenn der Senat eine notwendige Beweiserhebung anordnet. Sie beginnt dann mit Abschluß der Beweiserhebung. Kompetenzstreitigkeiten sind sehr selten und sind im Zeitraum der letzten Publikationen des Senates (Oktober 90-Juni 1991) nicht vorgekommen.

\section{Ausblick}

Trotz der relativ guten Inf rastruktur erscheint der Senat hof fnungslos überlastet. Dies liegt an der hohen Anzahl von zu entscheidenden Verfahren, die nur zum Teil für das Vertrauen der Bevölkerung in den Senat sprechen. Ein wichtiger Grund für die hohe Anzahl der Verfahren ist insbesondere die Schnelligkeit, mit der auf diese Weise eine Entscheidung zu erreichen ist. Die in der LJC vorgesehenen Fristen sind extrem kurz und der Zugang zum Senat sehr einfach. Außerdem darf man nicht vergessen, da $\beta$ in Costa Rica bei nur drei Millionen Einwohnern ca. fünftausend Anwälte tätig sind. Die Anzahl der Verfahren vor den Gerichten insgesamt ist sehr hoch und lag im Jahre 1993 über fünfhunderttausend (beim Verfassungssenat selber sind in dem Jahr 5355 Verfahren eingegangen). $\mathrm{Zu}$ bedenken ist weiterhin, daß der Senat erst seit Ende 1989 tätig ist und daher auch die Richtervorlagen und die "Acciones de Inconstitucionalidad" noch sehr zahlreich sind. Diese werden in der Zukunft wahrscheinlich abnehmen. Steigende Tendenz weisen dagegen die Habeas-Corpus-Verfahren und die Amparo-Verfahren auf. Eine kurzfristige Entlastung des Senates könnte durch seine Aufteilung in Kammern erreicht werden, die diese Fälle letztverbindlich entscheiden könnten. Derzeit muß jede Entscheidung, auch die Zurückweisung wegen offensichtlicher Unzulässigkeit, vom gesamten Senat getroffen werden. Die einzige Entscheidung, die der Präsident alleine treffen kann, die Annahme der "Acción de Inconstitucionalidad", kann mit einem Rechtsmittel angegriffen werden und damit vor den gesamten Senat gebracht werden. Die Richter verlieren somit viel

Art. 109 LJC: "Le corresponde a la Sala Constitucional resolver:

a) Los conflictos de competencia o atribuciones entre los Poderes del Estado, incluido el Tribunal Supremo de Elecciones, o entre cualquiera de ellos y la Controlaría General de la República.

b) Los conflictos de competencia o atribuciones constitucionales entre cualquiera de los Poderes $u$ órganos dichos y las entidades descentralizadas, municipalidades y otras personas de Derecho Público, o los de cualesquiera de éstas, entre sí." 
unnötige Zeit für die Erledigung einfacher Fälle. Langfristig wird nur die Einführung der Voraussetzung der Rechtswegerschöpfung in Verbindung mit einer Reform der wichtigsten Verfahrensrechte eine wirkliche Entlastung bringen ${ }^{74}$. Zu prüfen wäre auch, ob die Konvertierung anhängiger Amparo-Verfahren und Richtervorlagen in eine "Acción de Inconstitucionalidad" wie oben dargelegt, wirklich notwendig ist. Überflüssig erscheint auch die in Art. 12 LJC vorgesehene Ergänzung und Erklärung der Urteile durch den Senat auf Antrag einer der Parteien. Diese müßten in der Regel so verfaßt werden, daß sie keiner weiteren Erklärung bedürfen. Notwendig ist hingegen eine ständige Aktualisierung der Entscheidungssammlung. Diese befindet sich in den meisten Materien Anfang 1994 auf einem Stand von Ende 1991. Durch eine raschere Veröffentlichung der Rechtsprechung könnten einige Verfahren vermieden werden. Dies wurde insbesondere bei den Richtervorlagen bereits erkannt. Diese werden nunmehr getrennt und damit schneller veröffentlicht. Ein Grund für die Verzögerung der Publikationen sind in erster Linie mangelnde finanzielle Mittel und die Arbeitsüberlastung des Senates, der auch für die Publikationen verantwortlich zeichnet ${ }^{75}$. Ein weiterer Grund für verspätete Veröffentlichungen ist auch die Möglichkeit der Abgabe von abweichenden Voten, die begründet werden können (dissenting vote). Die überlasteten Richter lassen sich oft extrem viel Zeit für die Abgabe der Begründung. Hier sollte eine Frist für die Abgabe eingeführt werden, nach deren Ablauf das Urteil auch ohne das "dissenting vote" veröffentlicht werden kann. Eine schnellere Publikationsmöglichkeit könnte durch die Einrichtung einer Loseblattsammlung erreicht werden, in der die Urteile notfalls in Form von Fotokopien abgeheftet werden könnten. In Hinblick auf die Publikationen ist weiterhin erstaunlich, daß die Parteien namentlich in den Entscheidungssammlungen benannt werden und sogar im Gesamtregister alphabetisch geordnet nach Namen aufgeführt werden. Dies ist unter dem Gesichtspunkt des Datenschutzes nicht unbedenklich, auch wenn die Verfahren öffentlich sind.

Die oben aufgeführten kritischen Anmerkungen beinträchtigen die Gesamtarbeit des Senates nicht, die als sehr erfolgreich bezeichnet werden kann. Es ist gelungen, das Verfassungsbewußtsein der gesamten Justizverwaltung, aber auch der Bevölkerung insgesamt zu steigern. Sowohl die Legislative als auch die Exekutive berücksichtigen in verstärktem Maße die Verfassung beim Erlaß neuer Gesetze bzw. bei der Ausführung der Gesetze. Durch die Rechtsprechung des Senates fand eine erhebliche Modernisierung des Strafverfahrens statt. Ähnliches scheint im arbeitsrechtlichen Verfahren bevorzustehen. Das Ansehen der Justizverwaltung in Costa Rica ist, im Gegensatz zu dem in den meisten lateinamerikanischen Ländern, sehr hoch. Hierzu hat der Verfassungssenat erheblich beigetragen. 


\title{
ABSTRACTS
}

\section{Constitutional Reform in Argentina}

\author{
By Brigitte F.P. Lhoёst
}

In 1994 the Argentinian Constitution, dating from 1853, was considerably revised in order to meet the demands of the twenty-first century. Yet, the direct motive of this monumental project seems to be more trivial; President Menem's wish to be re-elected to office, a feat prohibited by the 1853 Constitution. This article aims to describe the constitutional reforms and to analyse their consequences for the future political and legal structure of the Argentinian State.

\section{The Constitutional Senate in Costa Rica}

\section{By Norbert Lösing}

Following the Continental-European example, Costa Rica introduced a concentrated and specialized constitutional jurisdiction in 1989. At this time a Constitutional Senate was installed in the Supreme Court. Thanks to the jurisdiction of this Senate the Constitution has gained influence and a great number of laws have been reconsidered and modernized. The "Law of Constitutional Jurisdiction" (Ley de la Jurisdicción Constitucional, LJC) offers for the individual a simple and fast access to the Senate which means fast and efficient legal protection. The terms in which the judges have to decide are very short. The fast and easy procedure are partly the reasons for the popularity of the Senate. The LJC does not require the exhaustion of other legal instruments to lodge a legal remedy before the Constitutional Senate. This is why a great number of litigants choose the direct access to the Senate instead of trying other legal remedies first. This tendency is supported by the Senate itself through his policy of accepting almost any case. Competencies of the Constitutional Senate are the habeas corpus, the amparo, the remedies of inconstitutionality and the conflicts of competence. In the search for exoneration of the Senate several possibilities are being discussed like introducing divisions or even returning to a diffuse constitutional control. 